\title{
Plasma Exchange Plus Mycophenolate Mofetil, Hormones with Severe Systemic Lupus Erythematosus Serum Autoantibodies
}

\author{
Shuai Ping,Guo Dang \\ Gannan Medical University,JiangXi GanZhou 341000 \\ 346591653@163.com
}

\begin{abstract}
Keywords:Plasmapheresis; mycophenolate mofetil; hormone; severe systemic lupus erythematosus; serum autoantibodies
\end{abstract}

\begin{abstract}
Analysis and Discussion plasmapheresis combined hormone, influence of mycophenolate mofetil on children with severe systemic lupus erythematosus autoantibodies. 80 cases in April 2013-Children with systemic lupus erythematosus in April 2015 in our hospital for treatment,in a random manner which were divided into two groups,namely: the control group and the observation group,the control group of patients children simple line of MMF combined hormone therapy in the treatment group,based on the observation of the patient group were treated with plasma exchange treatment,compared two groups of children with changes in the indicators and efficacy. The two groups of children after treatment,all patients anti-ds-DNA,SM antibody detection rate was significantly lower than before treatment, $\mathrm{P}<0.05$, group of children was lower than that observed in children in the control group, $\mathrm{P}<0.05$, anti-RNP antibody detection rate did not change significantly, $\mathrm{P}>0.05$. Compared with the previous treatment, were significantly increased in children with Cre children control group after treatment in the observation group, $24 \mathrm{~h}$ urine protein and ESR and other indicators, and the children of C3 decreased,compared with the control group,patients in the observation group the indicators improved obviously, $\mathrm{P}<0.05$. Patients observation group was $97.5 \%$, the effective treatment of patients in the control group was $82.5 \%$ in the observation group patients was significantly higher efficiency, $\mathrm{P}<0.05$, statistically significant.
\end{abstract}

\section{Introduction}

Systemic lupus erythematosus (systemic lupus ery thematosus, SLE) is a kind of autoimmune disease, the main features of T,B lymphocyte abnormalities, difficulty in treating large and complex pathogenesis ${ }^{[1]}$.In the past, treatment mainly cyclophosphamide,chloroquine,azathioprine corticosteroids and other treatment, although it is possible to control the patient's condition, however,it will increase the cancer,infections and bone marrow suppression and other dangerous diseases. To explore and research new therapeutic approaches and methods SLE, this study of plasma exchange hormone, mycophenolate mofetil (my cophenolate mofetil, MMF) treatment in children with severe systemic lupus erythematosus,a significant effect,the folloeing speciic reports:

\section{Materials and Methods}

\subsection{The clinical data}

Select 80 cases of 71 cases suffering from April 2013-Children with systemic lupus erythematosus in April 2015 in our hospital for treatment,all patients occurred fever, joint pain and other symptoms, of which 65 cases were typical facial erythema,children face edema, 19 cases of children with skin surface ulcer,22 cases of children with mental weakness, paleness, 18 cases of children with cough, severe symptoms of systemic lupus erythematosus in Figure 1.In a random 
manner which were divided into two groups, namely: the control group and the observation group,in which the children in the control group,21 males and 19 females,aged 4 to 16 years, median age was $(9.0 \pm 1.0)$ years, duration of 1 month to 2.5 years, with a median disease duration $(1.2 \pm 0.1)$ years;children with the observation group,23 males and 17 females, aged 3 to 17 years, median age was $(9.2 \pm 0.9)$ years,duration of 2 months to 2.8 years, with a median disease duration $(1.4 \pm 0.5)$ years. Two groups of children by sex, age and other general information is not obvious difference, $\mathrm{P}>0.05$, comparable.

\subsection{Treatment}

The control group of children with a simple line of MMF combined hormone therapy, specific treatment methods are:daily doses MMF1.5 2.0g treatment last about six months later,to reduce the dose of $0.75 \mathrm{~g} / \mathrm{d}$, while the addition of methylprednisolone hormone treatment, a daily dose of $0.5 \mathrm{~g}$, the impact of treatment after about $3 \mathrm{~d}$,for patients with oral prednisone therapy,patients treatment for 1.5 years.

In the control group,based on the observation group were added with plasma exchange treatment.Specific steps are:(1) configuration plasma replacement fluid.The blood:Colloid:Crystal= $1: 2: 2$ ratio to be configured, $1000 \mathrm{ml}$ liquid crystals, $500 \mathrm{ml}$ fresh frozen blood, $1000 \mathrm{ml} \%$ hydroxyethyl starch colloidal solution;(2) plasmapheresis.Before plasma exchange,we must ensure that children with stable heart rate and blood,platelets,WBC and other control in the normal range. Fresenius blood by machine,arteriovenous blood plasma separator line and the implementation of the replacement blood pressure.Blood volume replacement time should be maintained at 2 3h,replacement fluid input order colloidal $\sim$ crystals $\sim$ blood.

\subsection{OUTCOME MEASURES}

Joint pain for two groups of children before and after treatment,fever,edema, and rash were observed to improve the situation, the detection of children with liver and kidney function,blood,urine and changes in the frequency and extent of the occurrence of Raynaud's disease.Were observed before and after treatment of anti-RNP, anti-SM antibodies, expression of ESR (Equivalent Series Resistance, ESR),ds-DNA and C3, etc., while comparing two groups of patients before and after treatment $24 \mathrm{~h}$ creatinine (creatinine, Cre), urinary protein changes.

\subsection{The evaluation criteria}

Treatment of two groups of children were observed markedly:the children of the observer status indicators are normal; effective:children observe all indicators have reached the normal standard,and the clinical symptoms have abated;invalid:various clinical parameters were children improve,or even worsen the situation.

\subsection{The statistical analysis}

Using statistical software SPSS16.0 for statistical analysis, using mean difference \pm standard

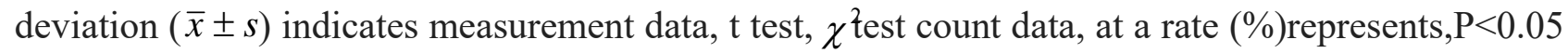
represents the difference more obvious, with statistically significance.

\section{Results}

\section{1 detection index between two groups of patients}

After treatment for two groups of children,all the children of anti-ds-DNA,SM antibody detection rate was significantly lower than before treatment, $\mathrm{P}<0.05$, group of children was lower than that observed in the control group of children, $\mathrm{P}<0.05$, anti-RNP antibody detection rate did not change significantly, $\mathrm{P}>0.05$, Table 1 . Compared with the previous treatment,were significantly increased in children with Cre children control group after treatment in the observation group,24h urine protein and ESR and other indicators, and the children of C3 decreased,compared with the 
control group,patients in the observation group the indicators improved obviously, $\mathrm{P}<0.05$, Table 2 .

Table 1: Comparison antibody detection rate of two groups of patients before and after treatment

\begin{tabular}{|c|c|c|c|c|c|}
\hline \multicolumn{6}{|c|}{$[n=40, n(\%)]$} \\
\hline \multirow[t]{2}{*}{ Group } & Anti-ds-DNA antibody & \multicolumn{2}{|c|}{ Anti-SM antibodies } & \multicolumn{2}{|c|}{ Anti-RNP antibody } \\
\hline & Before treatment After treatment & Before treatmen & After treatmen & Before treatment & t After treatmen \\
\hline Observer Group & $32(80.0) \quad 8(20.0) * \#$ & $38(95.0)$ & $9(22.5) * \#$ & $23(57.5)$ & $10(25.0) * \#$ \\
\hline Group & $31(77.5) 13(32.5)$ * & $39(97.5)$ & $15(37.5) *$ & $25(62.5)$ & $13(32.5) *$ \\
\hline
\end{tabular}

Note: Compared to before treatment, $\mathrm{P}<0.05$; compared with the control group, $\# \mathrm{P}<0.05$, statistically significant.

Table 2: Comparison of the indicators before and after treatment in two groups $(\mathrm{n}=40, \bar{x} \pm s)$

\begin{tabular}{|c|c|c|c|c|c|}
\hline \multirow{2}{*}{$\frac{\text { Group }}{\text { treatment }}$} & \multicolumn{2}{|c|}{$\operatorname{ESR}(\mathrm{mm} / \mathrm{h})$} & $\operatorname{Cre}(\mu \mathrm{mol} / \mathrm{L}$ & \multirow[t]{2}{*}{ complementC3(g/L) } & 24 hUrinary protein $(\mathrm{g})$ \\
\hline & Before & After & Before & & Before \\
\hline Observer Group & $92 \pm 24$ & $28 \pm 14 * \#$ & $512.4 \pm 39.8201 .8 \pm 30.3 * \#$ & $0.25 \pm 0.110 .65 \pm 0.14 * \#$ & $8.13 \pm 4.751 .84 \pm 1.54 * \#$ \\
\hline Group & $93 \pm 20$ & $52 \pm 11 *$ & $510.9 \pm 35.9398 .5 \pm 32.5^{*}$ & $0.25 \pm 0.12 \quad 0.40 \pm 0.01 *$ & $8.16 \pm 3.254 .52 \pm 1.50^{*}$ \\
\hline
\end{tabular}

Note: Compared to before treatment, $\mathrm{P}<0.05$; compared with the control group, \# $\mathrm{P}<0.05$, statistically significant.

\subsection{Comparative treatment groups were}

Patients observation group was $97.5 \%$, the effective treatment of patients in the control group was $82.5 \%$ in the observation group patients was significantly higher efficiency, $\mathrm{P}<0.05$, statistically significant (Table 3).

Table 3: Comparison treatment groups of patients [n (\%)]

\begin{tabular}{ccccc}
\hline Group & Significant effect & effective & No effect & Effective treatment \\
\hline Group $(\mathrm{n}=40)$ & $32(80.0)$ & $7(17.5)$ & $1(2.5)$ & $39(97.5)$ \\
Observer Group $(\mathrm{n}=40)$ & $20(50.0)$ & $13(32.5)$ & $7(17.5)$ & $33(82.5)$ \\
$\chi^{2}$ & 10.158 & 4.156 & 4.265 & 6.251 \\
$\mathrm{P}$ & $<0.05$ & $<0.05$ & $<0.05$ & $<0.05$ \\
\hline
\end{tabular}

\section{Discussion}

At this stage, in the treatment of clinical SLE patients,is relatively widely used glucocorticoids, however, because if the individual by glucocorticoid treatment in patients with SLE, the patient will be a series of adverse reactions, but also has lower treatment efficiency, therefore,for the effective treatment of patients with SLE, this study of plasma exchange corticosteroids, mycophenolate mofetil therapy for SLE patients ${ }^{[2]}$.

Methylprednisolone belongs to glucocorticoids, whose main role is anti-inflammatory, immunosuppressive, because glucocorticoid itself is the basis for the treatment of SLE disease drug, therefore,SLE patients usually take a long time to take glucocorticoids.And in cell immunity, glucocorticoid hormones relatively strong,there is an inhibitory role in the immune response to each phase of the cell immunity ${ }^{[3]}$, in particular,outstanding performance in the cellular immune function, if widely used, could significantly inhibit the body fluids of patients reaction,reduce antibody production, if the patient over a large number of glucocorticoids, dissolution occurs. 
The so-called plasmapheresis,in fact, through the blood separator replacement of serum immunoglobulin molecules, antibodies and immune complexes,in order to achieve the disease blood purification technology for therapeutic purposes, is currently in clinical plasma exchange has been a physicianrecognition of science and technology in recent years with the continuous development and progress,and gradually divided plasmapheresis selective and non-selective plasma exchange plasmapheresis and immune assay and other blood purification technology ${ }^{[4]}$. The study found that patients with plasma anti-ds-DNA antibodies,anti-SM antibodies and anti-RNP antibodies disappear or decline,clinical symptoms improved more obvious, shows that plasma exchange in the treatment of children with severe SLE aspect extremely efficacy exact. In addition,during plasmapheresis, must be carried out on the fluid balance in children strictly,total fresh and frozen blood colloid should not exceed $2 / 3$ of the total amount of liquid,if the claim is less than the amount of liquid can result in children with dizziness, headache and other symptoms.

MMF is a non-competitive,reversible and a new single nucleotide deoxy inosine inhibitor,in the past mainly used for kidney transplant rejection prevention and treatment in recent years usually MMF therapy clinical SLE,clinical the study found, MMF in the treatment of SLE is extremely significant aspect of the effect ${ }^{[5]}$.

The results of this study showed that patient a therapeutically effective observation group was $97.5 \%$, the treatment of patients in control group was $82.5 \%$ in the observation group patients was significantly higher efficiency, $\mathrm{P}<0.05$, statistically significant, we can see, plasma exchange plus MMF,steroid effective treatment of severe SLE,reduce serum autoantibodies,effective control of the disease in children, worthy of clinical application and promotion.

\section{References}

[1]Xu jian,Xiaoying Luo,Yi Ma,and other,plasma exchange combined with the clinical efficacy of drugs for children with severe systemic lupus erythematosus[J]Treatment of Clinical Research, 2013,30 (7):11-12.

[2]Haitao Zhang,Zhengzhao Liu,Weixin Hu,Clinical Observation double plasmapheresis combined hormone therapy of severe lupus nephritis[J]Journal of Nephrology Dialysis \& Transplantation, 2013,22 (3):163-164.

[3]Xiaoyan ta,Zian Liang,Aicheng Yang.Combined hormonal and immune adsorption efficacy of mycophenolate mofetil in patients with severe lupus nephritis observation[J]Guangxi Medicine, 2014,43(3):380-381.

[4]Yan Zhang,Xuan Xu,Ying Heand other.plasma exchange combined with continuous veno-venous hemofiltration one case of severe systemic lupus erythematosus[J]Chinese Pediatric Emergency Medicine, 2011,18(1):89-90.

[5]Min Zhang,Zheng Wang.children with systemic lupus erythematosus plasmapheresis clinical efficacy[J]western medicine,2011,23(5):920-921. 